\title{
STRUCTURAL INSTABILITY OF EXPONENTIAL FUNCTIONS
}

\author{
ZHUAN YE
}

\begin{abstract}
We first prove some equivalent statements on $J$-stability of families of critically finite entire functions. Then, with these in hand, a conjecture concerning stability of the family of exponential functions is affirmatively answered in some cases.
\end{abstract}

\section{INTRODUCTION}

In recent years, there have been many papers on the dynamics of some important classes of transcendental entire functions. The Julia set of a transcendental entire function is necessarily quite different from that of a polynomial or a rational map since the point at infinity is an essential singularity of transcendental entire function, although many results are true for both rational functions and transcendental entire functions.

One of the basic problems in iteration theory is to study structural stability of holomorphic families (for definitions, see $\S 1$ ). The simplest and the most interesting family is the exponential function family. A well-known conjecture (e.g. see [6]) in this field is that $\lambda e^{z}(\lambda \neq 0)$ is not structurally stable. In 1984, R. Devaney [4] proved that $e^{z}$ is not structurally stable. In 1989, J. Zhou and $\mathrm{Z}$. Li [11] proved that $\lambda e^{z}$ is unstable for any $\lambda>1 / e$. Here we show that $\lambda e^{z}$ is not structurally stable if $\lambda$ is on Cantor sets of curves tending to $\infty$ in the $\lambda$-plane, which is called the "Hairs" [3], (where the half line $\lambda>1 / e$ in the $\lambda$-plane is contained in the "hairs"), and other choices of $\lambda$. More interesting, the bifurcation diagram of $\lambda e^{z}$ in the $\lambda$-plane is known as Figure 1 (e.g. [1, 3, and 5]). Roughly speaking, for any $\lambda$ in the black area of Figure 1, we have $J\left(E_{\lambda}\right)=\mathbb{C}$ and for any $\lambda$ in the white area in Figure 1 , the $J\left(E_{\lambda}\right) \neq \mathbb{C}$. The black regions correspond to curves where the Julia set is the whole plane. The computer algorithm used to generate this picture simply colored a point if the corresponding exponential map satisfied $\operatorname{Re} E_{\lambda}^{n}(0)>100$ for some $n \leq 100$. However the main result in this paper shows that for any black $\lambda$, which lies on "hairs", there is a white $\lambda$ which can be arbitrarily close to the black $\lambda$. Of course, we cannot see this from the computer picture.

Received by the editors January 15, 1993 and, in revised form, September 29, 1993.

1991 Mathematics Subject Classification. Primary 58F23. 


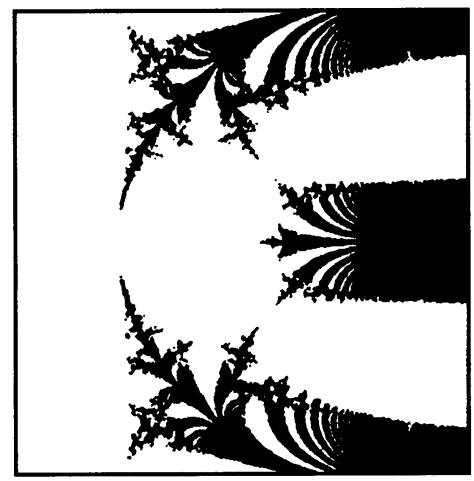

FIGURE $1[3]^{1}$. The topological structure of the bifurcation diagram of $\lambda e^{z}$.

\section{NOTATIONS AND PRELIMINARIES}

In the sequel we always regard $f$ as a transcendental entire function and denote the $n$-fold iterate of $f$ by $f^{n}$. Set

$$
F(f)=\left\{z:\left\{f^{n}\right\} \text { is normal in a neighborhood of } z\right\} .
$$

Then the Julia set of $f$ is $J(f)=\mathbb{C} \backslash F(f)$. A point $a \in \mathbb{C}$ is called periodic if $f^{n}(a)=a$ for some positive integer $n$. The minimal $n$ is called period of $a$, and $\left\{a, f(a), \ldots, f^{n-1}(a)\right\}$ is called a periodic cycle. If $a$ is an $n$ periodic point, we define $\lambda=\left(f^{n}\right)^{\prime}(a)$ to be the multiplier of $a$. The point $a$ is called attracting, repelling and neutral when $|\lambda|<1,|\lambda|>1$ and $|\lambda|=1$ respectively. Thus we can define the Julia set of any entire function $f$ as the closure of repelling periodic points. Moreover $J(f)$ is a perfect and completely invariant set. Standard references are [2] and [9] for rational functions and [6] for transcendental entire functions.

A point $a \in \mathbb{C}$ is called an asymptotic value of the entire function $f$ if there is a curve $\gamma \subset \mathbb{C}$ tending to $\infty$ such that $f(z) \rightarrow a$ as $z \rightarrow \infty$ and $z \in \gamma$. If $f^{\prime}(c)=0$, then $c$ is called a critical point of $f$ and $f(c)$ is called a critical value. Let

$$
\operatorname{sing} f^{-1}=\{\text { all critical values of } f\} \cup\{\text { all asymptotic values of } f\}
$$

be the set of singular points of $f$. Set

$$
S_{q}=\left\{f ; f \text { is entire and sing } f^{-1} \text { contains } q \text { points }\right\}, \quad S=\bigcup_{q=1}^{\infty} S_{q} .
$$

The $S$ is called the critically finite family. We call two entire functions $f(z)$ and $g(z)$ topologically equivalent in $\mathbb{C}$ if there are homeomorphisms $\phi, \psi: \mathbb{C}$ $\rightarrow \mathbb{C}$ such that $\psi \circ g=f \circ \phi$, and write $f \sim g$. For any nonconstant $g \in S_{q}$, set

$$
M_{g}=\{f ; f \text { is entire and } f \sim g\} .
$$

Clearly $M_{g}$ is a subset of $S_{q}$. Moreover $M_{g}[6, \S 3]$, is a $(q+2)$-dimensional complex analytic manifold and the topology of $M_{g}$ is locally equivalent to the

1 Reprinted from Julia sets and bifurcation diagrams for exponential maps, by R. Devaney, Bull. Amer. Math. Soc. 11 (1984), pp. 167-171 by permission of the American Mathematical Society. 
topology of uniform convergence on compact subsets of $\mathbb{C}$. In the sequel, we write a given submanifold of $M_{g}$ as simply $M$ for some fixed $g \in S$.

Let $M$ be such a submanifold. We define a multi-valued analytic function $\alpha_{p}: M \rightarrow \mathbb{C}$ as the set of solutions to the equation $f^{p}(\alpha)=\alpha$ (see [6] for full discussions). By [6, Theorem 2], the function $\alpha_{p}$ has only algebraic singularities. Let $\alpha_{p, i}(f)$ be a branch of $\alpha_{p}$. Set

$$
\begin{array}{rlrl}
N_{p} & =\left\{f \in M ;\left(f^{p}\right)^{\prime}\left(\alpha_{p, i}(f)\right) \text { for some } i\right\}, & \Sigma=M \backslash N, & \bar{M} \\
\lambda_{p}(f) & =\left(f^{p}\right)^{\prime}\left(\alpha_{p}(f)\right), & N=\bigcup_{p=1}^{\infty} N_{p} .
\end{array}
$$

Clearly $\lambda_{p}(f)$ is a multi-valued analytic function. We call an entire function $f_{0} \in M J$-stable in $M$ if for all $f \in M$ which are close enough to $f_{0}$, we have that $f_{0} \mid J\left(f_{0}\right)$ and $f \mid J(f)$ are topologically conjugate, i.e. there exists a homeomorphism $h_{f}: J\left(f_{0}\right) \rightarrow J(f)$ such that

$$
h_{f} \circ f_{0}(z)=f \circ h_{f}(z), \quad \text { for all } z \in J\left(f_{0}\right)
$$

and $h_{f}$ depends continuously on $f$ under the topology of uniform convergence on compact sets, $h_{f_{0}}=\mathrm{id}$ and $h_{f}$ is analytic as $f$ ranges in $M$. We call an entire function $f_{0} \in M$ structurally stable in $M$ if for all $f \in M$ which are close enough to $f_{0}$, we have that $f_{0}$ and $f$ are topologically conjugate in the whole complex plane and the conjugating homeomorphism depends continuously on $f$.

Definition. A holomorphic motion of a set $A \subset \mathbb{C}$ over $U$ (originating at $f_{0}$ ) is a map $h: U \times A \rightarrow \mathbb{C}$ satisfying the following conditions:

(1) The map $h(f, z)$ is analytic in $f$ for every $z \in A$,

(2) The map $h_{f}: z \mapsto h(f, z)$ is injective for every $f \in U$,

(3) $h_{f_{0}}=\mathrm{id}$.

\section{Statement of Results}

Let $\lambda_{0} \in \mathbb{C} \backslash\{0\}$ and $\delta$ be any positive real number.

$$
O_{\delta}\left(\lambda_{0}\right)=\left\{\lambda ;\left|\lambda-\lambda_{0}\right|<\delta\right\}, E_{\lambda}(z)=\lambda e^{z} \text { and } g_{n}(\lambda)=E_{\lambda}^{n}(0),
$$

where $E_{\lambda}^{n}$ is the $n$-fold iterate of $E_{\lambda}(z)$ and $\delta$ is so small that $O_{\delta}\left(\lambda_{0}\right) \cap\{0\}=$ $\varnothing$. One important question concerning complex dynamical systems (see, for example, $[3,6])$ asks:

Is there an open set of $\lambda$ in the $\lambda$-plane for which $J\left(E_{\lambda}\right)=\mathbb{C}$ ? (Or, is $J\left(E_{\lambda}\right)$ structurally stable whenever $J\left(E_{\lambda}\right)=\mathbb{C}$ ?)

Based on the classification of Sullivan for Fatou set, it is known that (e.g. Baker and Rippon [1], Devaney [3]) that

Theorem A. Let $\lambda_{0} \in \mathbb{C} \backslash\{0\}$.

$(*)$ If $g_{n}\left(\lambda_{0}\right) \rightarrow \infty \quad(n \rightarrow \infty)$, then $J\left(E_{\lambda_{0}}\right)=\mathbb{C}$.

(**) If $\lambda_{0}=k \pi i \quad(k \in \mathbb{Z})$, then $J\left(E_{\lambda_{0}}\right)=\mathbb{C}$.

In the sequel we say that $\lambda$ satisfies condition $(*)$ if $g_{n}\left(\lambda_{0}\right) \rightarrow \infty$ as $n \rightarrow \infty$ and condition $(* *)$ if $\lambda=k \pi i$, where $k \in \mathbb{Z}$. 
Remark. In [6, Theorem 8], Eremenko and Lyubich generalized Theorem A to critically finite families. They proved that if the orbits of all singular points of $f \in S$ land on cycles or tend to $\infty$ then $J(f)=\mathbb{C}$.

It is known that $E_{\lambda}(\lambda>1 / e)$ is not structurally stable [4] and [11]. In this paper we first prove some equivalent propositions of $J$-stability for the family of critically finite entire functions and specialize to the family $E_{\lambda}$, and then prove $E_{\lambda}$ is not structurally stable when $\lambda$ satisfies condition $(*)$ or condition $(* *)$. More precisely, we have

Theorem 1. Let $M$ be a submanifold of an $M_{g}$ as above. Then the following statements are equivalent:

(a) The period of the longest attracting cycle of $f \in M$ is bounded uniformly in $M$.

(b) For each $f \in M, f \in \Sigma$.

(c) $M$ is $J$-stable.

(d) Let $1 \leq i \leq q$, and choose $c_{i}(f) \in \operatorname{sing} f^{-1}$, and suppose that $c_{i}(f)$ is analytic in $M$ and $\mathscr{F}=\left\{f^{n}\left(c_{i}(f)\right)\right\}_{n=1}^{\infty}$ is an analytic family. Then $\mathscr{F}$ is a normal family.

Corollary 2. Let $\delta>0$ be fixed, then the following statements are equivalent.

(a) The period of the longest attracting cycle of $E_{\lambda}$ is bounded uniformly in $O_{\delta}\left(\lambda_{0}\right)$.

(b) For each $\lambda \in O_{\delta}\left(\lambda_{0}\right), E_{\lambda} \in \Sigma$.

(c) For any $\lambda \in O_{\delta}\left(\lambda_{0}\right)$, $E_{\lambda}$ is $J$-stable in $O_{\delta}\left(\lambda_{0}\right)$.

(d) $\left\{g_{n}(\lambda)\right\}$ is normal in $O_{\delta}\left(\lambda_{0}\right)$.

Theorem 3. If $\lambda_{0}$ satisfies the condition $(*)$, then $E_{\lambda_{0}}$ is not $J$-stable.

Corollary 4. Let $\lambda_{0}$ satisfy condition $(*)$. Then given $\varepsilon>0$, there exists a $\lambda_{*}$ such that $\left|\lambda_{0}-\lambda_{*}\right|<\varepsilon$ and $J\left(E_{\lambda_{*}}\right) \neq \mathbb{C}$; hence $E_{\lambda_{0}}$ is not structurally stable.

Theorem 5. If $\lambda_{0}$ satisfies the condition (**), then $E_{\lambda_{0}}$ is not $J$-stable.

Corollary 6. let $\lambda_{0}$ satisfy condition (**), then given $\varepsilon>0$, there exists $a \lambda_{*}$ such that $\left|\lambda_{0}-\lambda_{*}\right|<\varepsilon$ and $J\left(E_{\lambda_{*}}\right) \neq \mathbb{C}$; hence $E_{\lambda_{0}}$ is not structurally stable.

Remark. It seems that we cannot prove the most general case when 0 is preperiodic by using the methods in this paper, although we may generalize Corollary 6 a little by adding some restrictions.

I want to thank David Drasin, Alexandre Eremenko and Curt McMullen for suggestions and helpful discussions. Finally I want to thank Boston University for hospitality during the summer conference in dynamical systems in 1991.

\section{Proofs}

Proof of Theorem 1. We prove this theorem by combining the work of $\mathrm{C}$. McMullen [8] for rational mappings with that of A. Eremenko and M. Lyubich [6] for critically finite entire functions. Here we use the following procedure (a) $\Rightarrow$ (c) $\Rightarrow$ (d) $\Rightarrow$ (a) and (b) $\Leftrightarrow(c)$.

(a) $\Rightarrow$ (c) Suppose the period of the longest attracting cycle of any $f \in M$ is bounded uniformly in $M$. Then there exists an integer $p$ such that all $k$ periodic cycles of any $f \in M$ are repelling whenever $k \geq p$. In fact, suppose there is an $f$ which has a $k$-neutral cycle; i.e. $\left|\lambda_{k, i}(f)\right|=1$, for some branch 
$\lambda_{k, i}$ of $\lambda_{k}$. Since $\lambda_{k, i}(f)$ is a nonconstant analytic function [6, Lemma 6], there is an $f_{1} \in M$ which is close to $f$ with $\left|\lambda_{k}\left(f_{1}\right)\right|<1$. So $f_{1}$ has an attracting cycle with period $k \geq p$, and this contradicts (a). Now for any $f_{0} \in M$, let $U$ be a simply-connected neighborhood of $f_{0}$ in $M$. For any $k \geq p$, all branches $\alpha_{k, i}$ of $\alpha_{k}$ may be chosen, in a natural way, as singlevalued analytic functions in $U$ since $f_{0} \notin N_{k}$. Hence, as in [6, Theorem 9],

$$
h_{f}: U \times \operatorname{Per}_{p}\left(f_{0}\right) \rightarrow \mathbb{C} \quad \text { with } h_{f}\left(\alpha_{k, i}\left(f_{0}\right)\right)=\alpha_{k, i}(f)
$$

defines a holomorphic motion of the set of periodic points with period greater than $p$, which we write as $\operatorname{Per}_{p}\left(f_{0}\right)$, over $U$. (We denote the set of all periodic points of $f$ by $\operatorname{Per}(f))$. In fact, clearly $h_{f_{0}}=$ id and $h_{f}$ is analytic in $f$ as $f$ ranges in $U$ since $\alpha_{k, i}(f)$ is analytic. We next show that $h_{f}$ is injection for any fixed $f \in U$. If not so, there exist $f_{*} \in U, \alpha_{s, i}\left(f_{0}\right), \alpha_{t, j}\left(f_{0}\right)$ and $\alpha_{s, i}\left(f_{0}\right) \neq \alpha_{t, j}\left(f_{0}\right)$ such that $h_{f_{*}}\left(\alpha_{s, i}\left(f_{0}\right)\right)=\alpha_{s, i}\left(f_{*}\right)=\alpha_{t, j}\left(f_{*}\right)=h_{f_{*}}\left(\alpha_{t, j}\left(f_{0}\right)\right)$. Set $F_{s t}(f, z)=f^{s t}(z)-z$. So $F_{s t}\left(f_{*}, \alpha_{s, i}\left(f_{*}\right)\right)=F_{s t}\left(f_{*}, \alpha_{t}, j\left(f_{*}\right)\right)=0$ and $\frac{\partial}{\partial z} F_{s t}\left(f_{*}, \alpha_{s, i}\left(f_{*}\right)\right)=\left(f_{*}^{s t}\right)^{\prime}\left(\alpha_{s, i}\left(f_{*}\right)\right)-1 \neq 0$ and $\frac{\partial}{\partial z} F_{s t}\left(f_{*}, \alpha_{t, j}\left(f_{*}\right)\right) \neq 0$ since $f_{*} \in U$. Therefore applying the implicit function theorem to $F_{s t}(f, z)$ at $\left.\left(f_{*}, \alpha_{s, i} f_{*}\right)\right)=\left(f_{*}, \alpha_{t, j}\left(f_{*}\right)\right)$, we see that by the uniqueness of the solution to the equation $F_{s t}(f, z)=0$, there is a neighborhood of $f_{*}$, say $U^{\prime}$, such that $\alpha_{s, i}(f)=\alpha_{t, j}(f)$, for $f \in U^{\prime}$. Since these functions are analytic in $U$, they are equal to each other in $U$. Thus $\alpha_{s, i}\left(f_{0}\right)=\alpha_{t, j}\left(f_{0}\right)$, a contradiction.

It follows that the motion $h_{f}(z)$ is a holomorphic motion of $\operatorname{Per}_{p}\left(f_{0}\right)$ over $U$. By the $\lambda$-lemma [7], this motion can be extended to a holomorphic motion on $\overline{\operatorname{Per}_{p}\left(f_{0}\right)}$. However the set $\operatorname{Per}\left(f_{0}\right) \backslash \operatorname{Per}_{p}\left(f_{0}\right)$ has no limit point; otherwise we would have $f^{n}(z) \equiv z$, for some $n<p$. Hence $\operatorname{Per}_{p}\left(f_{0}\right)$ is dense in $J\left(f_{0}\right)$ since $\operatorname{Per}\left(f_{0}\right)$ is dense in $J\left(f_{0}\right)$ and $J\left(f_{0}\right)$ is perfect. Thus $\overline{\operatorname{Per}_{p}\left(f_{0}\right)}=J\left(f_{0}\right)$, and $h_{f}$ provides the conjugation which shows the $J$-stability of $f_{0}$. Hence $M$ is $J$-stable since $f_{0}$ was arbitrary.

To prove that (d) follows from (c), we need the following lemmas:

Lemma 1. Let $M$ be $J$-stable. If there exists $f_{0} \in M$ such that the forward orbit of some $c_{i_{0}}\left(f_{0}\right) \in \operatorname{sing} f_{0}^{-1}$ meets a repelling cycle of $f_{0}$, then for any $f \in M$, the forward orbit of $c_{i_{0}}(f)$ under $f$ must meet a repelling cycle of $f$.

Proof. Suppose the forward orbit of some $c_{i_{0}}\left(f_{0}\right)$ meets a repelling cycle $\left\{f_{0}^{m}\left(z_{0}\right)\right\}_{m=0}^{p-1}$; i.e. there exist $0 \leq n_{0} \leq p-1$ and $m$ such that $f_{0}^{n_{0}}\left(z_{0}\right)=$ $f_{0}^{m}\left(c_{i_{0}}\left(f_{0}\right)\right)$, thus $c_{i_{0}}\left(f_{0}\right) \in J\left(f_{0}\right)$. Since $M$ is $J$-stable, there is a neighborhood $U \subset M$ of $f_{0}$ such that for any $f \in U$, there exists a homeomorphism $h_{f}$ such that (1) holds. Hence we have that

$$
\left\{h_{f}\left(z_{0}\right), f \circ h_{f}\left(z_{0}\right), \ldots, f^{p-1} \circ h_{f}\left(z_{0}\right)\right\}
$$

is a repelling cycle of $f$. Furthermore the forward orbit of $h_{f}\left(c_{i_{0}}\left(f_{0}\right)\right)$ under $f$ meets this repelling cycle since

$$
f^{m} \circ h_{f}\left(c_{i_{0}}\left(f_{0}\right)\right)=h_{f} \circ f_{0}^{m}\left(c_{i_{0}}\left(f_{0}\right)\right)=h_{f} \circ f_{0}^{n_{0}}\left(z_{0}\right)=f^{n_{0}} \circ h_{f}\left(z_{0}\right), \quad f \in U .
$$

Moreover both sides of this equality are analytic in $M$, so it holds in all of $M$. In other words, for any $f \in M$ the forward orbit of $h_{f}\left(c_{i_{0}}\left(f_{0}\right)\right)$ meets a repelling cycle of $f$.

It remains to prove that $h_{f}\left(c_{i_{0}}\left(f_{0}\right)\right)=c_{i_{0}}(f)$ for $f \in M$, when $c_{i_{0}}\left(f_{0}\right) \in$ $J\left(f_{0}\right)$. Since $c_{i_{0}}\left(f_{0}\right) \in J\left(f_{0}\right)$, there exists a sequence $\left\{a_{k}\left(f_{0}\right)\right\} \subset \operatorname{Per}_{p}\left(f_{0}\right)$ such 
that $a_{k}\left(f_{0}\right) \rightarrow c_{i_{0}}\left(f_{0}\right) \quad(k \rightarrow \infty)$. Moreover, we can assume from the fact that $f_{0}$ is $J$-stable that there are a neighborhood of $f_{0}$, say $U$, and $\left\{a_{k}(f)\right\} \subset \operatorname{Per}_{p}\left(f_{0}\right)$ such that $a_{k}(f) \rightarrow c_{i_{0}}(f)$ for $f \in U$. By our definition of $h_{f}$, we have that $h_{f}\left(a_{k}\left(f_{0}\right)\right)=a_{k}(f)$, hence $h_{f}\left(c_{i_{0}}\left(f_{0}\right)\right)=c_{i_{0}}(f)$ So the lemma is proved.

Lemma 2. Let $c_{i}\left(f_{0}\right) \in \operatorname{sing} f_{0}^{-1}$. Then there exists a repelling cycle with period greater than 3 of $f_{0}$ such that the forward orbit of $c_{i}\left(f_{0}\right)$ does not meet this cycle.

Proof. Take any repelling cycle $\left\{f_{0}^{k}\left(z_{0}\right)\right\}_{k=0}^{p_{0}-1} \quad\left(p_{0} \geq 3\right)$ of $f_{0}$. If the forward orbit of $c_{i}\left(f_{0}\right)$ does not hit this cycle, then the lemma is proved. Otherwise, noting $f_{0}$ has infinitely many repelling cycles, we can find another repelling cycle (period greater than 3 ) which is disjoint on the orbit of $c_{i}\left(f_{0}\right)$. Thus the lemma is completely proved.

(c) $\Rightarrow$ (d) Suppose $M$ is $J$-stable. For any $1 \leq i \leq q$ and for fixed $f_{0} \in$ $M$, let $c_{i}\left(f_{0}\right) \in \operatorname{sing} f_{0}^{-1}$. Then by Lemma 2 , there exists a repelling cycle $\left\{f_{0}^{k}\left(z_{*}\right)\right\}_{k=0}^{p_{0}-1} \quad\left(p_{0} \geq 3\right)$ of $f_{0}$ such that the forward orbit of $c_{i}\left(f_{0}\right)$ under $f_{0}$ does not meet this repelling cycle; i.e. $\left\{f_{0}^{n}\left(c_{i}\left(f_{0}\right)\right)\right\}_{n=0}^{\infty} \cap\left\{f_{0}^{k}\left(z_{*}\right)\right\}_{k=1}^{p_{0}-1}=$ $\varnothing$. Thus we see from the proof of Lemma $1,\left\{f^{n}\left(c_{i}(f)\right)\right\}_{n=1}^{\infty}$ must avoid $\left\{h_{f}\left(f_{0}^{k}\left(z_{*}\right)\right)\right\}_{k=0}^{p-1}$, for all $f \in M$, where $h_{f}$ is from Lemma 1 . Set

$$
g_{n}^{i}(f)=\frac{f^{n}\left(c_{i}(f)\right)-h_{f}\left(z_{*}\right)}{f^{n}\left(c_{i}(f)\right)-h_{f}\left(f_{0}\left(z_{*}\right)\right)} \frac{h_{f}\left(f_{0}^{2}\left(z_{*}\right)\right)-h_{f}\left(f_{0}\left(z_{*}\right)\right)}{h_{f}\left(f_{0}^{2}\left(z_{*}\right)\right)-h_{f}\left(z_{*}\right)} .
$$

So $\left\{g_{n}^{i}(f)\right\}_{n=1}^{\infty}$ does not hit $0,1, \infty$ (since $h_{f}$ is one-to-one for each fixed $f \in M)$, and $g_{n}^{i}(f)$ is holomorphic in $M$. By Montel's theorem $\left\{g_{n}^{i}(f)\right\}_{n=1}^{\infty}$ is normal. Moreover set $a(f)=\left[h_{f}\left(f_{0}^{2}\left(z_{*}\right)\right)-h_{f}\left(f_{0}\left(z_{*}\right)\right)\right]\left[h_{f}\left(f_{0}^{2}\left(z_{*}\right)\right)-h_{f}\left(z_{*}\right)\right]^{-1}$, it follows from the fact $h_{f}\left(z_{*}\right) \neq h_{f}\left(f_{0}\left(z_{*}\right)\right)$ that $g_{n}^{i}(f) \neq a(f)$. Thus solving for $f^{n}$ in (2), we have that

$$
f^{n}\left(c_{i}(f)\right)=\frac{g_{n}^{i}(f) h_{f}\left(f\left(z_{*}\right)\right)-h_{f}\left(z_{*}\right) a(f)}{g_{n}^{i}(f)-a(f)} .
$$

Suppose that $g_{n_{j}}^{i}(f)$ tends to $b(f)$ on a compact subset of $M$. Then we have that either $b(f) \equiv a(f)$ or $b(f) \neq a(f)$, otherwise $g_{n_{j}}^{i}(f)-a(f)=0$ has solutions for all large $j$. It follows that $\mathscr{F}$ is normal. Thus (d) is proved.

(d) $\Rightarrow\left(\right.$ a) Suppose that the holomorphic family $\left\{f^{n}\left(c_{i}(f)\right)\right\}_{n=1}^{\infty}$ is normal in $M$ for each $i$. Let $F_{i}(f)$ denote thefunction which is the uniform limit of a subsequence of $\left\{f^{n}\left(c_{i} k(f)\right)\right\}_{n=1}^{\infty}$ in some compact subset of $M$. Now suppose for some $f_{0} \in M, f_{0}$ has an attracting cycle with some order $k$, say $\left\{f_{0}^{j}\left(z_{0}\right)\right\}_{j=0}^{k-1}$. (If not so, (a) is proved.) Thus this cycle must attract some $F_{i_{0}}\left(f_{0}\right)$, since each attracting basin contains a singular point of $f_{0}$, (e.g. see $[6, \S 5])$; i.e. $f_{0}^{k}\left(F_{i_{0}}\left(f_{0}\right)\right)=F_{i_{0}}\left(f_{0}\right)$. Hence $F_{i_{0}}(f) \neq \infty$. Applying the implicit function theorem to $\phi(f, z)=f^{k}(z)-z$ at the point $\left(f_{0}, z_{0}\right)$ and noting the continuity of $\lambda_{k}(f, z)=\left(f^{k}\right)^{\prime}(z)$, we find a neighborhood $U\left(f_{0}\right)$ such that any $f \in U\left(f_{0}\right)$ has an attracting cycle with period $k$. Since each attracting basin of $f$ contains a singular point of $f$ and the subsequence of the forward orbits of the singular point uniformly approaches the attracting cycle, we have that $f^{k}\left(F_{i_{0}}(f)\right)=F_{i_{0}}(f)$, for any $f \in U\left(f_{0}\right)$. Hence it holds in $M$. Thus $c_{i_{0}}(f)$ (the $i_{0}$ th singular point of $f$ ) is never attracted to a cycle with period greater 
than $k$ for all $f \in M$. Therefore if there is another $f_{1} \in M$ which has an attracting cycle with period $k_{1}>k$, then there is $c_{i_{1}}(f) \quad\left(i_{1} \neq i_{0}\right)$ such that $c_{i_{1}}(f)$ is attracted by a cycle with period no than $k_{1}$. However there are only finitely many singular points, so the longest length of an attracting cycle must be uniformly bounded.

Remark. Eremenko and Lyubich [6, Theorem 5] proved that if $f \in S_{q}$, then the number of attracting cycles is not greater than $q$. Hence the longest length of attracting cycle of $f$ is bounded.

(c) $\Rightarrow$ (b) Suppose $f_{0}$ is $J$-stable in $M$. Then there is a $U\left(f_{0}\right)$ in $M$ such that the number of repelling periodic cycles of any $f$ in $U\left(f_{0}\right)$ with period $p$ is constant, since $f_{0} \mid J\left(f_{0}\right)$ is topologically conjugate to $f \mid J(f)$ for any $f \in U\left(f_{0}\right)$ and for any integer $p$. Now we suppose that (b) is not true, i.e. $f_{0} \in N=$ $\overline{\bigcup_{p=1}^{\infty} N_{p}}$. If $f_{0} \in N_{p}$, for some $p$, then $\lambda_{p, i}\left(f_{0}\right)=1$ for some branch of $\lambda_{p}$. Since $\lambda_{p, i}(f)$ is a nonconstant analytic function [6, Lemma 6], there is a $f$ such that $f \in M, f$ arbitrarily close to $f_{0}$ such that the number of repelling cycles of $f$ with period $p$ is greater than that of $f_{0}$. This contradicts the assumption that the number of repelling cycles with period $p$ is constant in $U\left(f_{0}\right)$. Now we consider the case that $f_{0} \in N$ but $f_{0} \notin N_{p}$ for any $p$. Thus there exists a sequence $f_{n}$ such that $f_{n} \in \bigcup_{p=1}^{\infty} N_{p}$ and $f_{n}$ converges uniformly to $f_{0}$ on any compact subset. But the number of repelling cycles of order $p$ of any $f$ in $U\left(f_{0}\right)$ is constant for any fixed $p$. However we may take a $f_{n_{0}} \in N_{p_{0}} \cap U\left(f_{0}\right)$, so that $\lambda_{p_{0}, i}\left(f_{n}\right)=1$. As above there exists $f_{*} \in U\left(f_{0}\right)$ such that the number of repelling cycles with period $p_{0}$ of $f_{*}$ is greater than that of $f_{n_{0}}$ which in turn is equal to that of $f_{0}$. This again contradicts the fact that the number of repelling cycles with period $p$ is constant in $U\left(f_{0}\right)$. So we have proved the $(b)$.

(b) $\Rightarrow$ (c) See [6, Theorem 9].

Thus Theorem 1 is proved completely.

Proof of Corollary 2. We take $M=\left\{E_{\lambda} ; \lambda \in \Omega_{\delta}\left(\lambda_{0}\right)\right\}$. As in $\S 1$, let $\psi=$ id and $\phi=z+\log \left(\lambda_{1} / \lambda_{2}\right)$, we see that $E_{\lambda_{1}} \sim E_{\lambda_{2}}$ (for any $\lambda_{1}, \lambda_{2} \in O_{\delta}\left(\lambda_{0}\right)$ ). Hence $M$ is a submanifold of $S_{1}$. Furthermore the exponential family has only one asymptotic value 0 ; i.e. sing $E_{\lambda}^{-1}=\{0\}$ for all $\lambda \in O_{\delta}\left(\lambda_{0}\right)$. Hence $f^{n}(c(f))=E_{\lambda}^{n}(0)=g_{n}(\lambda)$. Thus the corollary follows from Theorem 1 .

Proof of Theorem 3. If $E_{\lambda_{0}}$ is $J$-stable, then there exists $\delta_{0}>0$ such that $M_{0}=\left\{E_{\lambda} ; \lambda \in O_{\delta_{0}}\left(\lambda_{0}\right)\right\}$ is $J$-stable. By Corollary 2, $\left\{g_{n}(\lambda)\right\}$ is normal in $O_{\delta_{0}}\left(\lambda_{0}\right)$. By hypothesis, $g_{n}\left(\lambda_{0}\right)=E_{\lambda_{0}}^{n}(0)$ tends to infinity, thus $g_{n}(\lambda)$ converges uniformly to infinity in $O_{\delta_{1}}\left(\lambda_{0}\right)$, where $\delta_{1} \leq \delta_{0}$. Without loss of generality we may assume $\delta=\delta_{0}=\delta_{1}$. Moreover, from the definition of $g_{n}(\lambda)$ we have that

$$
g_{n+1}(\lambda)=\lambda e^{g_{n}(\lambda)} .
$$

since $g_{n+1}$ tends uniformly to infinity, (3) implies that $\operatorname{Re} g_{n}(\lambda)$ also tends uniformly to $+\infty$. Now we consider two cases by looking at $\left\{g_{n}^{\prime}\left(\lambda_{0}\right)\right\}$.

Case One: $\left\{g_{n}^{\prime}\left(\lambda_{0}\right)\right\}$ is unbounded.

Choose a subsequence $n_{k}$ with $\left\{g_{n_{k}}^{\prime}\left(\lambda_{0}\right)\right\}$ tending to infinity $(k \rightarrow \infty)$. Set $h_{m}(\lambda)=g_{n}(\lambda)+\log \lambda$, for any fixed branch of logarithm. We may assume that each $h_{n}(\lambda)$ is analytic in $O_{\delta}\left(\lambda_{0}\right)$. Furthermore, given $M>1$, there is a $N_{1}$ such that $\left|h_{n_{k}}^{\prime}\left(\lambda_{0}\right)\right| \delta \geq 8 M\left(k \geq N_{1}\right)$. By applying Bloch's theorem 
to $\left\{h_{n_{k}}(\lambda)\right\}_{k=N_{1}}^{\infty}$ in $O_{\delta}\left(\lambda_{0}\right)$, we obtain that there is an open set $D_{n_{k}} \subset O_{\delta}\left(\lambda_{0}\right)$ such that $h_{n_{k}}\left(D_{n_{k}}\right)$ is a disk with $\operatorname{diam}\left(h_{n_{k}}\left(D_{n_{k}}\right)\right) \geq \sqrt{3}\left|h_{n_{k}}^{\prime}\left(\lambda_{0}\right)\right| \delta / 2 \geq 4 M$, $\left(k \geq N_{1}\right)$. Thus there exists $\lambda_{n_{k}}^{*} \in D_{n_{k}} \subset O_{\delta}\left(\lambda_{0}\right)$ and an integer $j_{n_{k}}$ such that $\operatorname{Im}\left(h_{n_{k}}\left(\lambda_{n_{k}}^{*}\right)\right)=\left(2 j_{n_{k}}+1 / 2\right) \pi,\left(k \geq N_{1}\right)$. So, if $\lambda_{n_{k}}^{*}=r_{n_{k}}^{*} e^{i \theta_{n_{k}}^{*}}$, we may arrange that $\operatorname{Im}\left(g_{n_{k}}\left(\lambda_{n_{k}}^{*}\right)\right)=\left(2 j_{n_{k}}+1 / 2\right) \pi-\theta_{n_{k}}^{*},\left(k \geq N_{1}\right)$. Hence, by (3),

$$
\begin{aligned}
\operatorname{Re} g_{n_{k}+1}\left(\lambda_{n_{k}}^{*}\right) & =\operatorname{Re}\left\{r_{n_{k}}^{*} e^{\operatorname{Re} g_{n_{k}}\left(\lambda_{n_{k}}^{*}\right)+i\left\{\operatorname{Im} g_{n_{k}}\left(\lambda_{n_{k}}^{*}\right)+\theta_{n_{k}}^{*}\right\}}\right\} \\
& =r_{n_{k}}^{*} e^{\operatorname{Re} g_{n_{k}}\left(\lambda_{n_{k}}^{*}\right)} \cos \left(\operatorname{Im} g_{n_{k}}\left(\lambda_{n_{k}}^{*}\right)+\theta_{n_{k}}^{*}\right) \\
& =0, \quad \text { for all } k \geq N_{1} .
\end{aligned}
$$

This contradicts the uniform convergence of $\left\{\operatorname{Re} g_{n}(\lambda)\right\}$ to infinity in $O_{\delta}\left(\lambda_{0}\right)$, so $E_{\lambda_{0}}$ is not $J$-stable and the theorem is proved in this case.

Case Two: $\left\{g_{n}^{\prime}\left(\lambda_{0}\right)\right\}$ is a bounded set. First we prove the following two claims.

Claim 1. If $\left\{g_{n}^{\prime}\left(\lambda_{0}\right)\right\}$ is bounded, then we have that $g_{n}^{\prime}\left(\lambda_{0}\right) \rightarrow-1 / \lambda_{0}$, as $n \rightarrow \infty$. Proof. If there is a subsequence $\left\{g_{n_{k}}^{\prime}\left(\lambda_{0}\right)\right\}$ with limit $a \neq-1 / \lambda_{0}$, then since $g_{n_{k}+1}^{\prime}\left(\lambda_{0}\right)=e^{g_{n_{k}}\left(\lambda_{0}\right)}\left(1+\lambda_{0} g_{n_{k}}^{\prime}\left(\lambda_{0}\right)\right), \quad \operatorname{Re} g_{n_{k}}\left(\lambda_{0}\right) \rightarrow+\infty$ and $1+\lambda_{0} g_{n_{k}}^{\prime}\left(\lambda_{0}\right) \rightarrow$ $1+\lambda_{0} a \neq 0$, we must have that $g_{n_{k}+1}^{\prime}\left(\lambda_{0}\right) \rightarrow \infty$, as $k \rightarrow \infty$. This contradicts the boundedness of $\left\{g_{n}^{\prime}\left(\lambda_{0}\right)\right\}$.

Claim 2. If $\left\{g_{n}^{\prime}\left(\lambda_{0}\right)\right\}$ is bounded, then there exists $\delta>0$ such that $g_{n}^{\prime}(\lambda) \rightarrow-1 / \lambda$ in $O_{\delta}\left(\lambda_{0}\right)$, as $n \rightarrow \infty$.

Proof. If there is a sequence $\lambda_{j}$ in $O_{\delta}\left(\lambda_{0}\right)$ with $\lambda_{j} \rightarrow \lambda_{0}$ and $\left\{g_{n}^{\prime}\left(\lambda_{j}\right)\right\}_{n=1}^{\infty}$ is unbounded for each $j$, then by Case One, for each $j, E_{\lambda_{j}}$ cannot be $J$-stable. It follows from the definition of $J$-stability of $E_{\lambda_{0}}$ that $E_{\lambda_{0}}$ is not $J$-stable. Hence there exists $\delta_{2}<\delta$ such that $\left\{g_{n}^{\prime}(\lambda)\right\}_{n=1}^{\infty}$ is bounded for each fixed $\lambda \in O_{\delta_{2}}\left(\lambda_{0}\right)$. By Claim 1 we must have $g_{n}^{\prime}(\lambda) \rightarrow-1 / \lambda$ in $O_{\delta_{2}}\left(\lambda_{0}\right)$. Again we assume $\delta_{2}=\delta$.

Now we want to get contradiction under the assumptions that $g_{n}^{\prime}(\lambda)$ tends to $-1 / \lambda$ in $O_{\delta}\left(\lambda_{0}\right)$ and that $g_{n}(\lambda)$ and $\operatorname{Re} g_{n}(\lambda)$ uniformly go to infinity and positive infinity respectively in $O_{\delta}\left(\lambda_{0}\right)$. Again we consider two cases.

Case (2.1). The convergence in Claim 2 is not uniform in any neighborhood of $\lambda_{0}$.

Case (2.2). The convergence in Claim 2 is uniform in some neighborhood of $\lambda_{0}$.

Case (2.1): Since $g_{n}^{\prime}(\lambda)$ converges to $-1 / \lambda$ and does not converge uniformly to $-1 / \lambda$ in any $O_{\delta}\left(\lambda_{0}\right)$, there exists a subsequence $\lambda_{n_{k}} \in O_{\delta}\left(\lambda_{0}\right)$ with $\lambda_{n_{k}} \rightarrow \lambda_{0}$ such that $g_{n_{k}}^{\prime}\left(\lambda_{n_{k}}\right)$ tends to a constant $b \neq-1 / \lambda_{0}$. If $b \neq \infty$, then $g_{n_{k}+1}^{\prime}\left(\lambda_{n_{k}}\right)=e^{g_{n_{k}}\left(\lambda_{n_{k}}\right)}\left(1+\lambda_{n_{k}} g_{n_{k}}^{\prime}\left(\lambda_{n_{k}}\right)\right)$ tends to infinity since $e^{g_{n_{k}}\left(\lambda_{n_{k}}\right)} \rightarrow \infty$ and $\left(1+\lambda_{n_{k}} g_{n_{k}}^{\prime}\left(\lambda_{n_{k}}\right)\right) \rightarrow\left(1+b \lambda_{0}\right) \neq 0$. So without loss of generality, we may assume $b=\infty$, i.e. $g_{n_{k}}^{\prime}\left(\lambda_{n_{k}}\right) \rightarrow \infty$. Thus there exist $\tilde{\lambda} \in\left\{\lambda_{n_{k}}\right\}_{k=1}^{\infty}$ and $N_{0}$ such that

$$
\left|g_{N_{0}}^{\prime}(\tilde{\lambda})\right| \geq 2 /|\tilde{\lambda}| \quad \text { and } \quad\left|g_{n}(\tilde{\lambda})\right| \geq 2, \quad\left(n>N_{0}\right) .
$$


Therefore we have, for all $n>N_{0}$,

$$
\begin{aligned}
\left|\tilde{\lambda} g_{n}^{\prime}(\tilde{\lambda})\right| & =\left|g_{n}(\tilde{\lambda})+g_{n}(\tilde{\lambda}) \tilde{\lambda} g_{n-1}^{\prime}(\tilde{\lambda})\right| \\
& =\left|g_{n}(\tilde{\lambda})+g_{n}(\tilde{\lambda}) g_{n-1}(\tilde{\lambda})+g_{n}(\tilde{\lambda}) g_{n-1}(\tilde{\lambda}) \tilde{\lambda} g_{n-2}^{\prime}(\tilde{\lambda})\right| \\
& =\mid g_{n}(\tilde{\lambda})+g_{n}(\tilde{\lambda}) g_{n-1}(\tilde{\lambda})+\cdots+g_{n}(\tilde{\lambda}) \cdots g_{N_{0}+1}(\tilde{\lambda}) \\
& \quad+g_{n}(\tilde{\lambda}) \cdots g_{N_{0}+1}(\tilde{\lambda}) \tilde{\lambda} g_{N_{0}}^{\prime}(\tilde{\lambda}) \mid \\
& \geq 2\left|g_{n} \cdots g_{N_{0}+1}(\tilde{\lambda})\right|-\left|g_{n} \cdots g_{N_{0}+1}(\tilde{\lambda})\right|-\cdots-\left|g_{n} g_{n-1}(\tilde{\lambda})\right|-\left|g_{n}(\tilde{\lambda})\right| \\
& \geq\left|g_{n}(\tilde{\lambda})\right| \rightarrow \infty, \quad \text { as } n \rightarrow \infty .
\end{aligned}
$$

This is impossible since by Claim $2, \tilde{\lambda} g_{n}^{\prime}(\tilde{\lambda}) \rightarrow-1$. Hence we have the theorem in Case (2.1).

Case (2.2): In this case we know that $g_{n}(\lambda)$ and $\operatorname{Re} g_{n}(\lambda)$ uniformly tend to $\infty$ and $+\infty$ respectively, and $g_{n}^{\prime}(\lambda)$ uniformly tends to $-1 / \lambda$ in $O_{\delta}\left(\lambda_{0}\right)$. Hence $g_{n}(\lambda)-g_{n}\left(\lambda_{0}\right)$ uniformly tends to $\log \left(\lambda_{0} / \lambda\right)$ in $O_{\delta}\left(\lambda_{0}\right)$. We may assume $\delta$ so small that $\log \left(\lambda_{0} / \lambda\right)$ is univalent in $O_{\delta}\left(\lambda_{0}\right)$. In the sequel, we regard $N_{0}$ as a sufficiently large number whose value each time may not be the same. By the Koebe's distortion theorem [10], for $n>N_{0}$ and $\lambda \in O_{\delta}\left(\lambda_{0}\right)$, we have

$$
\begin{aligned}
& \left|g_{n}^{\prime}\left(\lambda_{0}\right)\right|\left|\lambda-\lambda_{0}\right|\left(1+\lambda-\lambda_{0} \mid \delta^{-1}\right)^{-2} \\
& \quad \leq\left|g_{n}(\lambda)-g_{n}\left(\lambda_{0}\right)\right| \leq\left|g_{n}\left(\lambda_{0}\right)\right|\left|\lambda-\lambda_{0}\right|\left(1-\left|\lambda-\lambda_{0}\right| \delta^{-1}\right)^{-2} .
\end{aligned}
$$

Since $g_{n}^{\prime}\left(\lambda_{0}\right) \rightarrow-1 / \lambda_{0}$, we have that $g_{n}\left(O_{\delta}\left(\lambda_{0}\right)\right) \quad\left(n>N_{0}\right)$ contains a disk with center $g_{n}\left(\lambda_{0}\right)$ whose radius $\eta_{0}$ is uniformly bounded from 0 .

Set $\lambda_{0}=r_{0} e^{i \theta_{0}}$ with $0 \leq \theta_{0}<2 \pi$, let $\gamma\left(\theta_{0}\right)$ be the radial segment, i.e.

$$
\gamma\left(\theta_{0}\right)=\left\{\lambda ; \lambda=r e^{i \theta_{0}} \in O_{\delta}\left(\lambda_{0}\right), r_{0}-\delta \leq r \leq r_{0}+\delta\right\},
$$

We obtain from (5) that

(6) $\min \left\{\left|g_{n}\left(\left(r_{0}+\delta\right) e^{i \theta_{0}}\right)-g_{n}\left(\lambda_{0}\right)\right|,\left|g_{n}\left(\left(r_{0}-\delta\right) e^{i \theta_{0}}\right)-g_{n}\left(\lambda_{0}\right)\right|\right\}>\delta / 8\left|\lambda_{0}\right| \equiv r_{*}$,

for all large $n$. Since $g_{n}(\lambda)-g_{n}\left(\lambda_{0}\right) \rightarrow \log \left(\lambda_{0} / \lambda\right)$ we have that

$$
\operatorname{Re} g_{n}\left(\left(r_{0}+\delta\right) e^{i \theta_{0}}\right)<\operatorname{Re} g_{n}\left(\lambda_{0}\right)<\operatorname{Re} g_{n}\left(\left(r_{0}-\delta\right) e^{i \theta_{0}}\right), \quad\left(n>N_{0}\right) .
$$

Now $\log \left(\lambda_{0} / \lambda\right)$ maps $\gamma\left(\theta_{0}\right)$ to a horizontal line segment on real axis, so that $g_{n}(\lambda)=\log \left(\lambda_{0} / \lambda\right)+g_{n}\left(\lambda_{0}\right)+\varepsilon_{n}(\lambda)$ (where $\varepsilon_{n}(\lambda)$ tends uniformly to zero) maps $\gamma\left(\theta_{0}\right)$ to a curve segment $\Delta_{n}$ which for large $n$ is asymptotic to a horizontal segment line passing through $g_{n}\left(\lambda_{0}\right)$. However since the exponential function maps a horizontal line to a ray starting at the origin, the equality $g_{n+1}(\lambda)=$ $r e^{i \theta_{0}} e^{g_{n}(\lambda)}, \lambda \in \gamma\left(\theta_{0}\right)$, tells us that the image $g_{n+1}\left(\gamma\left(\theta_{0}\right)\right)$ is a curve segment $\gamma_{n+1}$ which tends to the line segment contained in the ray $\Gamma_{n+1}=\left\{t e^{i\left(\operatorname{Im}_{g_{n}}\left(\lambda_{0}\right)+\theta_{0}\right)}\right.$, $t>0\} \quad\left(n>N_{0}\right)$. Hence the two curve segments $\Delta_{n}$ and $\gamma_{n}$ must be close when $n>N_{0}$. Since $e^{R e_{g n}(\lambda)}$ is large for $n>N_{0}$, this means that if $\lambda \in \gamma\left(\theta_{0}\right)$ then $\operatorname{Im} g_{n}(\lambda)+\theta_{0}=2 k_{n} \pi+\varepsilon_{n}$, where $k_{n}=k_{n}\left(\theta_{0}\right)$ is an integer and $\varepsilon_{n} \rightarrow$ 0 . Therefore $\left(\operatorname{Im} g_{n}(\lambda)+\theta_{0}\right)(\bmod 2 \pi)$ converges uniformly to zero in $\gamma\left(\theta_{0}\right)$ since $\operatorname{Im} g_{n}(\lambda)+\operatorname{Im} g_{n}\left(\lambda_{0}\right)$ tends uniformly to 0 in $\gamma\left(\theta_{0}\right)$ and $\left(\operatorname{Im} g_{n}(\lambda)+\theta_{0}\right)$ $(\bmod 2 \pi)$ tends to zero.

Moreover using (6), (7), the fact that $\operatorname{Im} g_{n}\left(\lambda_{0}\right) \rightarrow-\theta_{0}(\bmod 2 \pi)$ and that $\Delta_{n}$ is close to a segment of $\gamma_{n}$, we may take a point $\lambda_{n} \in \gamma\left(\theta_{0}\right)$ such that $\operatorname{Im}_{g_{n}}\left(\lambda_{n}\right)+\theta_{0}=0(\bmod 2 \pi)$. Thus $\operatorname{Im} g_{n+1}\left(\lambda_{n}\right)=0$. So if $\theta_{0} \neq 0$, this 
contradicts the fact that $\operatorname{Im} g_{n}(\lambda)(\bmod 2 \pi)$ tends uniformly to $-\theta_{0}$ in $\gamma\left(\theta_{0}\right)$. However if $\theta_{0}=0$, then $\lambda_{0}$ must be real. Moreover $E_{\lambda_{0}}^{n}(0) \rightarrow \infty$ implies that $\lambda_{0}>1 / e$. It can be easily seen that $q_{n}^{\prime}\left(\lambda_{0}\right)$ must tend to infinity, which has already been discussed in Case One in this case. Thus Theorem 3 is completely proved.

Proof of Corollary 4. By Theorem 3, we know that $E_{\lambda_{0}}$ is not $J$-stable. Hence there is a $\delta<\varepsilon$ such that the family $\left\{\lambda e^{z} ;\left|\lambda-\lambda_{0}\right|<\delta\right\}$ is not $J$-stable. But $J$-stability is equivalent to structural stability since $J\left(E_{\lambda_{0}}\right)=\mathbb{C}$. It follows from Corollary 2(b) that there is a $\lambda_{*} \in O_{\delta}\left(\lambda_{0}\right)$ such that $E_{\lambda_{*}} \notin \Sigma$. Therefore $J\left(E_{\lambda_{*}}\right) \neq \mathbb{C}$ since $J\left(E_{\lambda_{0}}\right)=\mathbb{C}$.

Remark 1. By [3], we know that there are uncountable unions of curves tending to $\infty$ in the $\lambda$-plane such that for all $\lambda$ except on the endpoints of these curves, $E_{\lambda}^{n}(0) \rightarrow \infty$.

Remark 2. If $\lambda_{0}>0$, then both $g_{n}\left(\lambda_{0}\right)$ and $g_{n}^{\prime}\left(\lambda_{0}\right)$ are positive. So

$$
g_{n}^{\prime}\left(\lambda_{0}\right)=e^{g_{n-1}\left(\lambda_{0}\right)}+g_{n}\left(\lambda_{0}\right) g_{n-1}^{\prime}\left(\lambda_{0}\right) \geq e^{g_{n-1}\left(\lambda_{0}\right)} \rightarrow \infty, \quad(n \rightarrow \infty) .
$$

Hence by Theorem 3 (Case One) and Corollary 4, we have an alternate proof of [4 and 11].

Proof of Theorem 5. We first prove the case $\lambda_{0}=2 k \pi i \quad(k \neq 0$ is a given integer). Then we have $E_{\lambda_{0}}(0)=2 k \pi i=E_{\lambda_{0}}^{2}(0)$; i.e. $g_{2}\left(\lambda_{0}\right)=\lambda_{0}=g_{1}\left(\lambda_{0}\right)$, so, $g_{n}\left(\lambda_{0}\right)=\lambda_{0},(n>0)$. As in the proof of Theorem 3, if $E_{\lambda_{0}}$ is $J$-stable, it follows from Corollary 2(d) that there exists $\delta>0$ such that $\left\{g_{m}(\lambda)\right\}=\left\{E_{\lambda}^{m}(0)\right\}$ is normal in $O_{\delta}\left(\lambda_{0}\right)$. Hence there exists a subsequence $\left\{g_{m_{j}}(\lambda)\right\}$ which necessarily converges to a holomorphic function $g(\lambda)$ in $O_{\delta / 2}\left(\lambda_{0}\right)$ since $g_{n}\left(\lambda_{0}\right)=\lambda_{0}$, for all $n>0$. Thus $\left\{g_{m_{j}}^{\prime}\left(\lambda_{0}\right)\right\}$ is bounded since $g^{\prime}\left(\lambda_{0}\right)$ is finite. However from (3) we have that

$$
\lambda g_{n}^{\prime}(\lambda)=g_{m}(\lambda)+g_{m}(\lambda) \lambda g_{m-1}^{\prime}(\lambda), \quad \text { for all } m>0 .
$$

Hence we obtain using $(8)$ that $\left(\lambda_{0} \neq 0\right)$,

$$
\begin{aligned}
\lambda_{0} g_{m}^{\prime}\left(\lambda_{0}\right)= & g_{m}\left(\lambda_{0}\right)+g_{m}\left(\lambda_{0}\right) g_{m-1}\left(\lambda_{0}\right)+\cdots \\
& +g_{m}\left(\lambda_{0}\right) \cdots g_{2}\left(\lambda_{0}\right)+g_{m}\left(\lambda_{0}\right) \cdots g_{2}\left(\lambda_{0}\right) \lambda_{0} g_{1}^{\prime}\left(\lambda_{0}\right) \\
= & \lambda_{0}+\lambda_{0}^{2}+\cdots+\lambda_{0}^{m-1}+\lambda_{0}^{m} \\
= & \lambda_{0}\left(\lambda_{0}^{m}-1\right) /\left(\lambda_{0}-1\right) \rightarrow \infty .
\end{aligned}
$$

This contradicts the boundedness of the $\left\{g_{m_{j}}^{\prime}\left(\lambda_{0}\right)\right\}$, and Theorem 5 is proved in this case.

Next set $\lambda_{0}=2(k+1) \pi i \quad\left(k\right.$ a fixed integer). Then $E_{\lambda_{0}}^{2}(0)=-2(k+1) \pi i=$ $E_{\lambda_{0}}^{3}(0)$. Set $a=E_{\lambda_{0}}^{2}(0)$. We have that $E_{\lambda_{0}}^{n}(0)=a$ and $g_{n}\left(\lambda_{0}\right)=a,(n \geq 2)$. Hence if the theorem is not true for this $\lambda_{0}$, then there exists $\delta>0$ such that $\left\{g_{m}(\lambda)\right\}=\left\{E_{\lambda}^{m}(0)\right\}$ is normal in $O_{\delta}\left(\lambda_{0}\right)$. Therefore there exists a subsequence such that $\left\{g_{m_{j}}^{\prime}\left(\lambda_{0}\right)\right\}$ is a bounded set since $g_{n}\left(\lambda_{0}\right)=a(n \geq 2)$. However, 
again we have that

$$
\begin{aligned}
\lambda_{0} g_{m}^{\prime}\left(\lambda_{0}\right)= & g_{m}\left(\lambda_{0}\right)+g_{m}\left(\lambda_{0}\right) g_{m-1}\left(\lambda_{0}\right)+\cdots \\
& +g_{m}\left(\lambda_{0}\right) \cdots g_{2}\left(\lambda_{0}\right)+g_{m}\left(\lambda_{0}\right) \cdots g_{2}\left(\lambda_{0}\right) \lambda_{0} g_{1}^{\prime}\left(\lambda_{0}\right) \\
= & a+a^{2}+\cdots+a^{m-1} a^{m-1} \lambda_{0} \\
= & \frac{a}{1-a}-a^{m} \frac{2-a}{1-a} \rightarrow \infty .
\end{aligned}
$$

Therefore as in the paragraph above we have a contradiction. Thus the theorem is completely proved.

Proof of Corollary 6. The proof is quite similar to that of Corollary 4.

\section{REFERENCES}

1. I. N. Baker and P. J. Rippon, Iteration of exponential functions, Ann. Acad. Sci. Fenn. 9 (1984), 49-77.

2. A. F. Beardon, Iteration of rational functions, Graduate Texts in Math., vol. 132, SpringerVerlag, 1991.

3. R. Devaney, Julia sets and bifurcation diagrams for exponential maps, Bull. Amer. Math. Soc. 11 (1984), 167-171.

4. $\longrightarrow$, The structural instability of $\exp (z)$, Proc. Amer. Math. Soc. 94 (1985), 545-548.

5. R. Devaney and M. Krych, Dynamics of $\exp (z)$, Ergodic Theory Dynamical Systems 4 (1984), 35-52.

6. A. Eremenko and Yu. M. Lyubich, Dynamical properties of some classes of entire functions, Ann. Sci. Ecole Norm. Sup. 42 (1992).

7. R. Mañe, P. Sad, and D. Sullivan, On the dynamics of rational maps, Ann. Sci. Ecole Norm. Sup. 16 (1983), 192-217.

8. C. McMullen, Families of rational maps and iterative root-finding algorithms, Ann. of Math. (2) 125 (1987), 467-494.

9. J. Milnor, Dynamics in one complex variable, SUNY at Stony Brook \#5 preprint, 1990.

10. G. Valiron, Fonctions analytiques, Presses Universitaires de France, Paris, 1954.

11. J. Zhou and Z. Li, structural instability of mapping $z \rightarrow \lambda \exp (z)\left(\lambda>e^{-1}\right)$, Sci. China Ser. A 30 (1989), 1153-1161.

Department of Mathematical Sciences, Northern Illinois University, DeKalb, IlliNOIS 60115

E-mail address: ye@math.niu.edu 